\title{
Ischemic stroke and depression
}

\author{
DAVID W. DESMOND,${ }^{1}$ ROBERT H. REMIEN,${ }^{2}$ JOAN T. MORONEY, ${ }^{3}$ YAAKOV STERN, ${ }^{4}$ \\ MARY SANO, ${ }^{4}$ AND JANET B.W. WILLIAMS ${ }^{2}$ \\ ${ }^{1}$ Departments of Neurology and Pathology, SUNY Downstate Medical Center, Brooklyn, New York \\ ${ }^{2}$ Department of Psychiatry, Columbia University College of Physicians and Surgeons, New York, New York \\ ${ }^{3}$ Department of Clinical Neurosciences, Royal College of Surgeons in Ireland, Beaumont Hospital, Dublin, Ireland \\ ${ }^{4}$ Departments of Neurology and Psychiatry and the Gertrude H. Sergievsky Center, Columbia University College of Physicians \\ and Surgeons, New York, New York
}

(Received February 20, 2002; Revised April 29, 2002; ACCEPTED May 28, 2002)

\begin{abstract}
Previous studies of depression after stroke have reported widely variable findings, possibly due to differences between studies in patient characteristics and methods for the assessment of depression, small sample sizes, and the failure to examine stroke-free reference groups to determine the base rate of depression in the general population. In an effort to address certain of those methodologic issues and further investigate the frequency and clinical determinants of depression after stroke, we administered the Structured Interview Guide for the Hamilton Depression Rating Scale (SIGH-D) and neurological, neuropsychological, and functional assessments to 421 patients (age $=71.5 \pm 8.0$ years) 3 months after ischemic stroke and 249 stroke-free control subjects (age $=70.8 \pm 6.7$ years). We required a SIGH-D total score $>11$ for the identification of depression. We found that depression was less frequent (47/421 patients, or $11.2 \%$, and 13/249 control subjects, or 5.2\%), less severe, and less persistent in our stroke cohort than previously reported, possibly due to the underrepresentation of patients with a premorbid history of affective illness. Depression was associated with more severe stroke, particularly in vascular territories that supply limbic structures; dementia; and female sex. SIGH-D item analyses suggested that a reliance on nonsomatic rather than somatic symptoms would result in the most accurate diagnoses of depression after ischemic stroke. (JINS, 2003, 9, 429-439.)
\end{abstract}

Keywords: Stroke, Cerebrovascular disease, Depression, Dementia

\section{INTRODUCTION}

Given that ischemic stroke is associated with a variety of adverse outcomes, including dementia (Desmond et al., 2000), dependent living (Pohjasvaara et al., 1998a), and death (Sarti et al., 2000), it is reasonable to presume that many stroke patients will experience significant depression. Although a number of prior studies have suggested that depression is a common consequence of stroke (Kotila et al., 1998; Pohjasvaara et al., 1998b; Robinson et al., 1983), that it is associated with specific lesion locations (Robinson et al., 1983, 1984; Starkstein et al., 1987, 1989), and that it may have an adverse impact on cognitive function (Bolla-Wilson et al., 1989), physical recovery (Parikh et al.,

Reprint requests to: Dr. David W. Desmond, SUNY Downstate Medical Center, 450 Clarkson Avenue, Box 25, Brooklyn, NY 11203. E-mail: dwdesmond@usa.net
1990), and survival (Morris et al., 1993), the results of studies of the frequency, determinants, and consequences of post-stroke depression have been remarkably variable when considered as a group (Gordon \& Hibbard, 1997). Certain methodologic features may have contributed to that variability, including differences between studies in patient characteristics and methods for the assessment of depression, small sample sizes, and the failure to examine stroke-free reference groups to determine the base rate of depression in the general population.

As a component of a prospective study of stroke and dementia (Desmond et al., 2000), we administered a standardized depression assessment to a large cohort of well-characterized ischemic stroke patients as well as a stroke-free age-matched reference group. Our goal in this subproject was to answer five specific questions: (1) What is the frequency of depression after ischemic stroke? (2) How do older subjects with and without stroke differ with regard 
to the frequency of the conventionally accepted symptoms of depression? (3) What are the clinical determinants of depression among ischemic stroke patients? (4) Is depression associated with dementia after stroke? and (5) How do stroke patients with and without dementia differ with regard to the frequency of the conventionally accepted symptoms of depression? We hypothesized that (1) stroke patients would meet criteria for depression more frequently than control subjects but less frequently than previously reported; (2) stroke patients would report depressed mood as well as a variety of somatic symptoms more frequently than control subjects; (3) depression among stroke patients would be associated with the severity of neurologic impairment but not lesion location; and (4) stroke patients with dementia would meet criteria for depression more frequently than nondemented patients, primarily due to a higher frequency of somatic symptoms rather than depressed mood in the former group.

\section{METHODS}

\section{Research Participants}

We recruited 585 subjects among patients consecutively admitted to Columbia-Presbyterian Medical Center for ischemic stroke. We recruited 297 of those patients from 1988 to 1990 and the remaining 288 patients from 1994 to 1997 , those two recruitment phases corresponding to two funding cycles. Eligibility requirements included an age 60 years or older and a diagnosis of ischemic stroke within the previous 30 days confirmed by brain imaging (relevant infarct or normal). When brain imaging was normal, the diagnosis of ischemic stroke was based on the patient's clinical history and the results of the neurologic examination (e.g., a presentation that includes the acute onset of a left hemiparesis that persists for at least $24 \mathrm{hr}$ ). Patients were permitted to be of either sex and any race or ethnicity and patients with a history of prior stroke or prior psychiatric illness were eligible for inclusion.

Patients were excluded when certain clinical features precluded a reliable assessment of cognitive function, including a Boston Diagnostic Aphasia Examination (Goodglass \& Kaplan, 1983) severity rating under 3 (lower scores represent greater severities of aphasia), impairment of consciousness persisting beyond the permitted 30-day poststroke enrollment period, or a primary language other than English or Spanish. Additional exclusion criteria included the presence of a concomitant neurologic disorder potentially affecting cognitive function (e.g., Parkinson's disease) or a severe comorbid medical illness (e.g., terminal cancer) that would preclude follow-up throughout the fiveyear duration of this prospective study. Patients were not excluded if a premorbid history of functional impairment suggested that they might have concomitant Alzheimer's disease because our larger study was designed to investigate both cerebrovascular disease and Alzheimer's disease as the two most frequent causes of dementia in the general population. Among patients who were otherwise eligible for inclusion, the most common reasons for exclusion from our study were the presence of neurologic deficits that were too severe to permit reliable and valid assessments (approximately 33\% of those who were excluded), refusal of enrollment by the patient and/or the patient's family (approximately 33\% of those who were excluded), and the presence of a severe comorbid medical illness that would preclude follow-up throughout the 5-year duration of this study (approximately 15\% of those who were excluded).

We also recruited a control cohort of 249 subjects (age $=$ $70.8 \pm 6.7$ years; education $=12.3 \pm 4.6$ years) who were free of any history of stroke or evidence of stroke in neurologic examination. Similar to the approach that we took to the recruitment of our stroke cohort, control subjects were not excluded if a premorbid history of functional impairment suggested that they might have Alzheimer's disease. Our focus upon stroke as the primary distinguishing feature between the stroke and control cohorts thus permits the direct assessment of that variable as a risk factor for depression, independent of other potentially confounding variables. Regarding additional demographic characteristics in our control cohort, $65.9 \%$ of the subjects were women, and $32.5 \%$ were Black, $14.9 \%$ were Hispanic, and $52.6 \%$ were White. The majority of the control cohort was randomly selected from the surrounding community based on a Medicare list, while the remaining subjects were spouses of stroke patients also enrolled in our study, who we recognize were likely to be at increased risk of depression themselves, or neighborhood volunteers who came to our attention due to advertisements or referrals by friends. As a group, control subjects were matched to the stroke cohort by age.

This study was approved by the Institutional Review Board of Columbia-Presbyterian Medical Center and all subjects provided informed consent.

\section{General Examination Procedures}

We performed our initial assessments 7 to 10 days after stroke. All patients were administered the Mini-Mental State Examination (MMSE; Folstein et al., 1975), a popular mental status screening test, and the Barthel Index (Mahoney \& Barthel, 1965), which taps the physical aspects of activities of daily living. Neurologists specializing in stroke administered a structured neurologic examination and documented any history of stroke, transient ischemic attack, or exposure to risk factors for cerebrovascular disease based on review of medical records and a structured interview administered to all patients and knowledgeable informants. Consistent cigarette use (i.e., at least 1 cigarette per day for at least 1 year) and consistent alcohol use (i.e., at least 1 serving of beer, wine, or hard liquor per week for at least 6 months) were also documented. A comprehensive medical history was recorded and any past psychiatric illnesses reported by the patient or a reliable informant or recorded in the patient's medical records were noted. Patients were also 
rated on the Stroke Data Bank Stroke Severity Scale (Foulkes et al., 1988). As part of routine clinical care, most of the patients recruited as part of our first cohort underwent a CT scan while most of the patients recruited as part of our second cohort underwent an MRI. In virtually all cases, brain imaging was performed on the day of admission, with follow-up scans typically performed when the initial scan did not demonstrate a relevant lesion. Based upon the review of clinical features and brain imaging studies, patients were classified by infarct location and stroke syndrome using a modification of the methods of the Stroke Data Bank. Characterization of the stroke syndrome was based upon the severity of the patient's neurologic deficits (e.g., major $v s$. minor) and the location of the infarction (e.g., left $v s$. right hemisphere).

Three months after stroke, all patients were administered the Structured Interview Guide for the Hamilton Depression Rating Scale (SIGH-D; Williams, 1988) and readministered the MMSE, the Barthel Index, the neurologic examination, and the Stroke Severity Scale. A comprehensive battery of neuropsychological tests developed for use in epidemiologic studies of dementia (Stern et al., 1992) was also given at that time. That battery was administered in either English or Spanish, based upon the language spoken in the subject's home, and it included measures of verbal and nonverbal memory (the Selective Reminding Test and a multiple-choice recognition version of the Benton Visual Retention Test), orientation (the Mini-Mental State Examination orientation subtest), language (a 15-item version of the Boston Naming Test, letter and category fluency tests, and selected items drawn from the repetition and complex ideation subtests of the Boston Diagnostic Aphasia Examination), visuospatial function (the Rosen Drawing Test and a multiple-choice matching version of the Benton Visual Retention Test), verbal and nonverbal abstract reasoning skills (the Similarities subtest of the Wechsler Adult Intelligence Scale-Revised and the Identities and Oddities subtest of the Mattis Dementia Rating Scale), and attention (cancellation tasks using shapes and letters as targets). Knowledgeable informants were administered the Blessed Functional Activity Scale (BFAS; Blessed et al., 1968), which taps the cognitive aspects of activities of daily living. Dementia was diagnosed based on criteria modified from the Diagnostic and Statistical Manual of Mental Disorders, Third Edition, Revised (DSM-III-R; American Psychiatric Association, 1987) and consistent with criteria later proposed by the International Workshop of the National Institute of Neurological Disorders and Stroke-Association Internationale pour la Recherche et l'Enseignement en Neurosciences (Román et al., 1993). Essentially, we required deficits in memory and two or more additional cognitive domains as determined in the neuropsychological evaluation as well as functional impairment not solely related to physical disability documented with the BFAS.

All available and willing patients were then seen for annual follow-up examinations during which all of the above assessments were repeated. Virtually all patients were hos- pitalized when they were assessed 7 to 10 days after stroke and virtually all patients were seen in our outpatient clinic when they were assessed three months and annually after stroke. Most of the 3-month post-stroke assessments were performed in the morning, although we did not require that they be performed at a specific time of day as part of our protocol.

\section{Assessment of Depression}

As noted above, we used the SIGH-D for the identification of depression. We selected the SIGH-D because the Hamilton Depression Rating Scale (HDRS; Hamilton, 1960, 1967) has been one of the most popular scales in studies of post-stroke depression, it is administered in a structured format, and it is not unduly demanding for use with stroke patients. In the SIGH-D, questions are posed to patients with regard to the presence or absence of the conventionally accepted symptoms of depression during the preceding week and follow-up questions are provided to elicit information that is directly relevant to scoring. The first question is, "What has your mood been like this past week?", for example, and it is followed by additional inquiries including, "Have you been feeling down or depressed?" and "Have you been crying at all?" Items are scored according to the frequency and intensity of depressive symptoms. Scoring options for that first question are zero points for the absence of depression, 1 point for depression that is indicated only on questioning, 2 points for depression that is spontaneously reported verbally, 3 points for depression that is communicated nonverbally (e.g., facial expression, tendency to weep), and 4 points for depression that is essentially the only focus of spontaneous verbal and nonverbal communication. All items were rated without assumptions regarding causation.

A cutoff score of 16 has been used in nonstroke samples based on the original version of the HDRS, but stroke studies have typically used lower cut-off scores (Andersen et al., 1994a). Trichotomous cut-off scores of over 24 for severe depression, 18 to 24 for moderate depression, 7 to 17 for mild depression, and zero to 6 for no depression have also been proposed (Endicott et al., 1981). Since no consensus exists on the optimal cut-off score to use for the identification of depression in a stroke sample, we used an intermediate cut-off of a score over 11 for that purpose in our study. Given that concerns have been expressed regarding the problematic influence of somatic scale items on the diagnosis of depression after stroke (Stein et al., 1996), we also performed secondary analyses in which we additionally required a score over 1 on the first SIGH-D item, which represents the spontaneous acknowledgment of depressed mood. In most cases, the SIGH-D was administered by research assistants, who were trained and supervised by a clinical psychologist (R.H.R.) on a continuing basis. When patients were considered to be unreliable, their responses on the SIGH-D were corroborated with reliable informants, when available. Neurologists were blind to the re- 
sults of the depression assessments when they performed their clinical examinations and reviewed brain imaging studies.

Studies of the reliability and validity of the SIGH-D and the HDRS in stroke samples are worthy of note. In our own work (reviewed in Desmond, 2000), three trained research assistants and one clinical psychologist administered the SIGH-D to 32 elderly ischemic stroke patients and 20 elderly stroke-free control subjects. Intraclass correlation coefficients were excellent for the 17-item total score (i.e., .96 for all raters and .97 for the three research assistants) and all but 2 of the 17 individual items (i.e., observer ratings of psychomotor retardation and agitation), including items related to depressed mood and anhedonia (i.e., .86 and .87, respectively). The results were comparable when data collected from the stroke and control groups were examined separately. Malec et al. (1990) reported an interrater reliability of .77 for the HDRS based on a Pearson correlation, with the second assessment administered within 11 days of the first assessment. Regarding the validity of the HDRS in stroke samples, Pearson correlations of .77, .74, and .70 have been reported for the association between HDRS total score and scores on the Geriatric Depression Scale, the Center for Epidemiologic Studies Depression Scale, and the Zung Self-Rating Depression Scale, respectively, in stroke patients (Agrell \& Dehlin, 1989). Other studies of patients with stroke have found that the HDRS total score increases with the severity of depression ( $\mathrm{Ng}$ et al., 1995) and decreases in association with the effective treatment of depression (Andersen et al., 1994b).

\section{Statistical Analyses}

To determine whether any selection bias might have resulted from our failure to examine a subset of patients 3 months after stroke, chi-square analyses were performed to compare the patients who were not examined to those patients who were examined with regard to the location, severity, and clinical manifestations of the presenting stroke; vascular risk factors; psychiatric history; and demographic and social variables. We then performed a logistic regression analysis to determine whether any of the variables found to be related to the failure to be examined 3 months after stroke in the univariate analyses $(p<.10)$ would be independently related to the failure to be examined.

We next performed a logistic regression analysis to characterize the risk of depression in the stroke versus the control cohort. To investigate the clinical determinants of depression, we performed chi-square analyses to compare the pooled group of patients who met criteria for depression to those who did not meet criteria for depression with regard to the clinical variables summarized above. We then performed a logistic regression analysis to determine whether any of the variables found to be relevant in the univariate analyses $(p<.10)$ would be independently related to depression status. To investigate the SIGH-D items that best distinguished stroke patients from control subjects and demented patients from nondemented patients, we performed chi-square analyses to compare each of those pairs of groups with regard to the frequency of positive responses to each SIGH-D item. We then performed logistic regression analyses to determine whether any of the SIGH-D items found to be related to stroke or dementia status in the univariate analyses $(p<.10)$ would be independently related to stroke or dementia status.

All logistic regression analyses were based on a backward stepwise variable removal method, with the criterion for variable removal set at $p \geq .10$. We selected that relatively liberal criterion rather than $p<.05$ because we sought to identify even weakly related variables, particularly those recognized in our primary analysis of the clinical determinants of depression that might be amenable to intervention.

\section{RESULTS}

\section{Clinical Characteristics of Patients Lost to Follow-Up}

Of the 585 patients who met our eligibility criteria and were initially enrolled in our study, 421 patients $(72.0 \%$; age $=71.5 \pm 8.0$ years) were examined 3 months after ischemic stroke. Of the 164 patients who were not examined, 26 patients had died, 40 patients were medically ill or too severely impaired neurologically to be assessed, and 98 patients refused follow-up, had moved, or had been lost to follow-up. Thus, of the 519 patients who could potentially have been examined, $421(81.1 \%)$ were actually examined. Patients who were not examined 3 months after stroke were significantly more impaired than patients who were examined on the MMSE $(20.5 \pm 6.3$ vs. $23.4 \pm$ 5.1), the Barthel Index $(58.3 \pm 33.1$ vs. $70.9 \pm 27.1)$, and the Stroke Severity Scale $(7.6 \pm 3.0$ vs. $6.6 \pm 3.1)$ administered 7 to 10 days after stroke based on $t$ tests. Chisquare analyses suggested that the failure to be examined 3 months after stroke was associated with the stroke syndrome, the location of the index stroke, congestive heart failure, atrial fibrillation, and age. Given that the stroke syndrome variable contained numerous response options, we recoded it into a dichotomous variable (i.e., major dominant and major nondominant syndromes versus all other syndromes) and found that a major hemispheral stroke syndrome, representing more severe neurologic impairment resulting from an infarction involving the left or right cerebral hemisphere, was associated with the failure to be examined three months after stroke. Logistic regression determined that the failure to be examined was independently associated with a major hemispheral stroke syndrome (odds ratio, OR: 1.67; 95\% confidence interval, CI: 1.07-2.61), congestive heart failure (OR: 1.66; CI: .982.83 ), and age 80 or older (OR: 2.52; CI: $1.54-4.13$ ) and 70 to 79 (OR: 1.61; CI: $1.04-2.47$ ) versus 60 to 69.

\section{Frequency and Severity of Depression After Ischemic Stroke}

Three-month post-stroke SIGH-D total scores stratified by the spontaneous acknowledgment of depressed mood (i.e., 
a SIGH-D first item score $>$ 1) are presented in Figure 1. Although the mean SIGH-D total scores of both the stroke and control cohorts were relatively low, clinically similar, and far below our cut-off for the identification of depression, the total score of the stroke cohort was significantly higher than the total score of the control cohort by $t$ test $(4.9 \pm 4.8$ vs. $3.5 \pm 3.9, p<.001)$. Forty-seven of the 421 stroke patients $(11.2 \%)$ and 13 of the 249 control subjects $(5.2 \%)$ received a SIGH-D total score over 113 months after stroke, yielding an odds ratio of 2.52 (CI: 1.33-4.80) for depression associated with stroke, adjusting for female sex (OR: 2.17; CI: 1.20-3.92). Thirty-one of the 421 patients $(7.4 \%)$ and 10 of the 249 control subjects $(4.0 \%)$ received a SIGH-D total score over 11 and spontaneously acknowledged depressed mood, yielding an odds ratio of 2.07 (CI: 0.99-4.35), adjusting for female sex (OR: 2.22; CI: $1.08-4.56)$.

Among the 47 patients who met criteria for depression 3 months after stroke, 4 patients $(8.5 \%)$ were taking anti-depressant medications, 6 patients $(12.8 \%)$ were taking anxiolytic medications, and 3 patients $(6.4 \%)$ were taking anti-psychotic medications. Among the 374 patients who did not meet criteria for depression three months after stroke, 21 patients $(5.6 \%)$ were taking anti-depressant medications, 15 patients $(4.0 \%)$ were taking anxiolytic medications, and 3 patients $(0.8 \%)$ were taking anti-psychotic medications.

\section{Clinical Determinants of Depression After Ischemic Stroke}

Within the stroke cohort, patients meeting criteria for depression 3 months after stroke were significantly more impaired than nondepressed patients on the MMSE (22.3 \pm 5.9 vs. $24.9 \pm 4.6)$, the Barthel Index (73.9 $\pm 31.9 v s$. $87.0 \pm 21.4$ ), and the Stroke Severity Scale (6.8 $\pm 3.1 \mathrm{vs}$. $5.1 \pm 3.2)$ administered at that time based on $t$ tests. Demographic and social characteristics stratified by depression status are shown in Table 1, vascular risk factors and psychiatric history stratified by depression status are shown in Table 2, and stroke characteristics stratified by depression status are shown in Table 3. Chi-square analyses suggested that depression was associated with dementia, a major hemispheral stroke syndrome, infarcts in the pooled anterior ce-

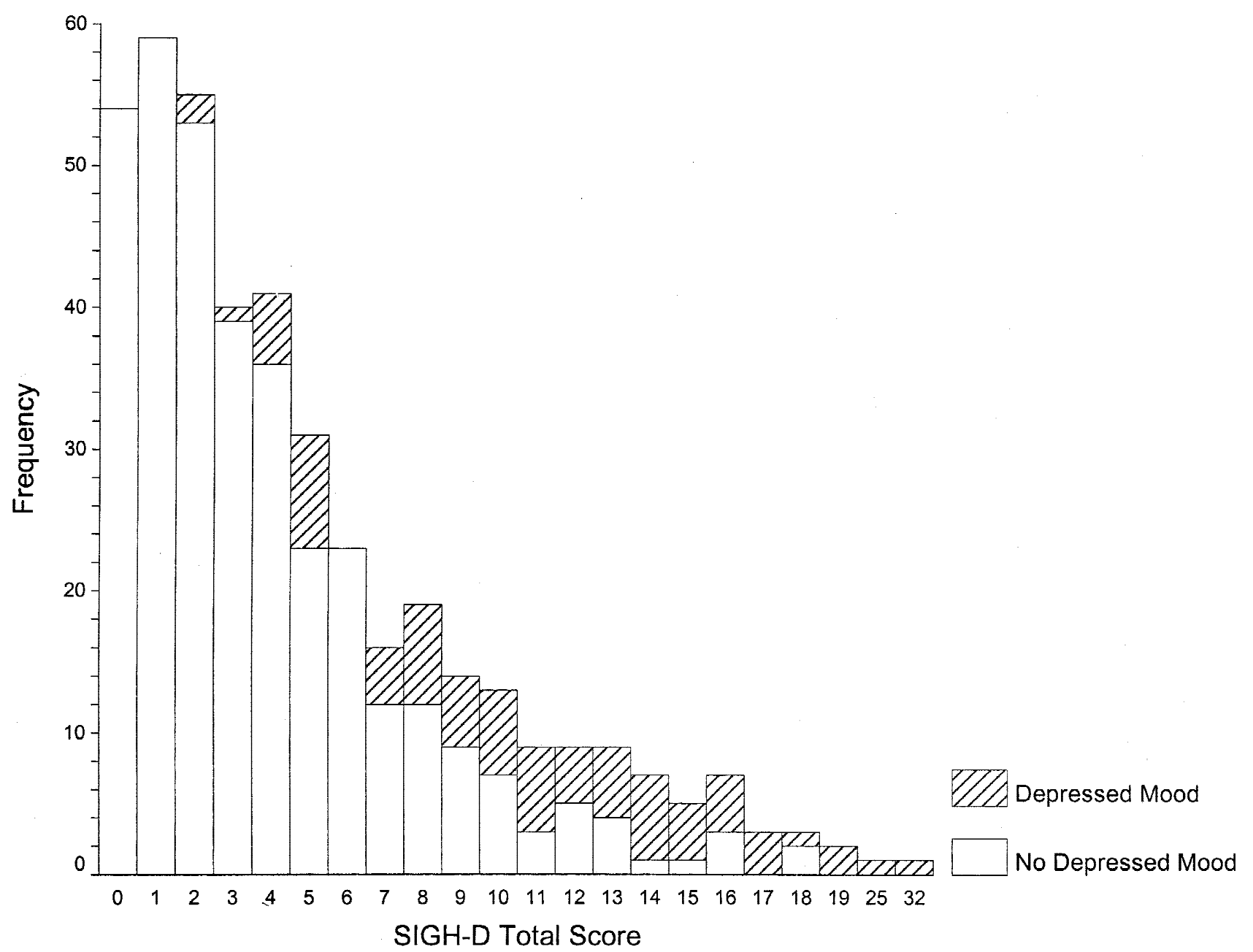

Fig. 1. Structured Interview Guide for the Hamilton Depression Rating Scale total score stratified by the spontaneous acknowledgment of depressed mood in the stroke cohort. 
Table 1. Demographic and social variables by depression status

\begin{tabular}{lrrr}
\hline \hline & \multicolumn{2}{c}{ Depression } & \\
\cline { 2 - 3 } & $\begin{array}{c}\text { Yes } \\
\text { Variable }\end{array}$ & $\begin{array}{c}\text { No } \\
(n=47)\end{array}$ & \\
\hline Age & & & \\
$\quad \geq 80$ years & $7(14.9)$ & $62(16.6)$ & .643 \\
$70-79$ years & $20(42.6)$ & $133(35.6)$ & \\
$\quad 60-69$ years & $20(42.6)$ & $179(47.9)$ & \\
Education & & & \\
0-8 years & $17(36.2)$ & $136(36.4)$ & .872 \\
9-12 years & $20(42.6)$ & $147(39.3)$ & \\
$\quad \geq 13$ years & $10(21.3)$ & $91(24.3)$ & \\
Ethnicity/race & & & \\
$\quad$ Black & $15(32.6)$ & $146(39.8)$ & .173 \\
Hispanic & $21(45.7)$ & $117(31.9)$ & \\
$\quad$ White & $10(21.7)$ & $104(28.3)$ & \\
Sex (female) & $31(66.0)$ & $188(50.3)$ & .042 \\
Predominant language (English) & $26(55.3)$ & $235(63.0)$ & .306 \\
Occupation (unskilled) & $24(51.1)$ & $204(54.8)$ & .624 \\
Handedness (right) & $45(97.8)$ & $345(93.0)$ & .209 \\
Residence (northern Manhattan) & $22(46.8)$ & $176(47.1)$ & .974 \\
Living alone at stroke onset & $16(34.0)$ & $128(34.5)$ & .950 \\
\hline \hline
\end{tabular}

Note. Figures are frequencies and within-group percentages. Significance levels are based on chi-square analyses. Some within-group percentages are based on an incomplete sample due to small amounts of missing data.

rebral artery (ACA) and posterior cerebral artery (PCA) territories versus infarcts in other vascular territories, a Barthel Index score of 80 or less, and sex. Logistic regression determined that depression 3 months after stroke was independently associated with dementia (OR: 3.18; CI: $1.65-$ 6.14), a major hemispheral stroke syndrome (OR: 1.68; CI: .80-3.53), infarcts in the pooled ACA and PCA territories versus infarcts in other vascular territories (OR: 1.64; CI: .78-3.48), and female sex (OR: 1.67; CI: .86-3.23). A

Table 2. Vascular risk factors and psychiatric history by depression status

\begin{tabular}{lrrr}
\hline \hline & \multicolumn{2}{c}{ Depression } & \\
\cline { 2 - 3 } & \multicolumn{1}{c}{ Yes } & \multicolumn{1}{c}{ No } & \\
Variable & $(n=47)$ & $(n=374)$ & $p$ \\
\hline Hypertension & $32(68.1)$ & $274(73.5)$ & .435 \\
Diabetes mellitus & $18(38.3)$ & $126(33.7)$ & .530 \\
Myocardial infarction & $8(17.0)$ & $64(17.2)$ & .975 \\
Angina & $13(27.7)$ & $82(22.2)$ & .397 \\
Atrial fibrillation & $5(10.6)$ & $49(13.2)$ & .625 \\
Congestive heart failure & $6(12.8)$ & $38(10.2)$ & .595 \\
Hypercholesterolemia & $12(25.5)$ & $85(23.0)$ & .703 \\
Consistent cigarette use & $25(53.2)$ & $219(59.2)$ & .432 \\
Consistent alcohol use & $22(46.8)$ & $186(50.3)$ & .655 \\
Prior stroke & $9(19.6)$ & $93(25.0)$ & .418 \\
Prior transient ischemic attack & $5(10.9)$ & $63(17.1)$ & .281 \\
History of psychiatric illness & $4(8.5)$ & $20(5.4)$ & .381 \\
\hline \hline
\end{tabular}

Note. Figures are frequencies and within-group percentages. Significance levels are based on chi-square analyses. Some within-group percentages are based on an incomplete sample due to small amounts of missing data.
Table 3. Stroke characteristics by depression status

\begin{tabular}{|c|c|c|c|}
\hline \multirow[b]{2}{*}{ Variable } & \multicolumn{2}{|c|}{ Depression } & \multirow[b]{2}{*}{$p$} \\
\hline & $\begin{array}{c}\text { Yes } \\
(n=47)\end{array}$ & $\begin{array}{c}\text { No } \\
(n=374)\end{array}$ & \\
\hline \multicolumn{4}{|l|}{ Stroke syndrome } \\
\hline Major dominant hemispheral & $7(14.9)$ & $27(7.2)$ & 095 \\
\hline Major nondominant hemispheral & $7(14.9)$ & $31(8.3)$ & \\
\hline Minor dominant hemispheral & $3(6.4)$ & $54(14.4)$ & \\
\hline Minor nondominant hemispheral & $5(10.6)$ & $53(14.2)$ & \\
\hline Lacunar/deep hemispheral & $19(40.4)$ & $129(34.5)$ & \\
\hline Brainstem/cerebellar & $6(12.8)$ & $80(21.4)$ & \\
\hline \multicolumn{4}{|l|}{ Stroke location } \\
\hline Left hemisphere & $17(36.2)$ & $124(33.2)$ & .598 \\
\hline Right hemisphere & $19(40.4)$ & $135(36.2)$ & \\
\hline Brainstem/cerebellum & $11(23.4)$ & $114(30.6)$ & \\
\hline Frontal location of presenting stroke & $10(21.3)$ & $81(21.7)$ & .952 \\
\hline Vascular territory (ACA or PCA $v s$. other) & $12(25.5)$ & $57(15.2)$ & .072 \\
\hline \multicolumn{4}{|l|}{ Stroke mechanism } \\
\hline Large artery atherosclerosis & $11(23.4)$ & $74(19.8)$ & 438 \\
\hline Cardiac embolism & $6(12.8)$ & $76(20.3)$ & \\
\hline Lacunar & $21(44.7)$ & $135(36.1)$ & \\
\hline Unknown/other cause & $9(19.1)$ & $89(23.8)$ & \\
\hline Barthel Index $(\leq 80)$ & $17(37.0)$ & $89(23.9)$ & .055 \\
\hline Dementia & $23(50.0)$ & $76(20.5)$ & $<.001$ \\
\hline
\end{tabular}

Note. Figures are frequencies and within-group percentages. Significance levels are based on chi-square analyses. Some within-group percentages are based on an incomplete sample due to small amounts of missing data. $\mathrm{ACA}=$ anterior cerebral artery; $\mathrm{PCA}=$ posterior cerebral artery.

further logistic regression analysis determined that depression with the spontaneous acknowledgement of depressed mood was independently associated with dementia (OR: 2.60; CI: 1.16-5.82), a major hemispheral stroke syndrome (OR: 2.34; CI: .99-5.50), infarcts in the pooled ACA and PCA territories versus infarcts in other vascular territories (OR: 2.50; CI: 1.07-5.84), angina (OR: 1.78; CI: .774.09), and female sex (OR: 1.70; CI: .75-3.84).

\section{Depression During Long-Term Follow-Up}

In our stroke cohort, 336 of the 421 patients $(79.8 \%)$ had at least 1 follow-up examination. Specifically, 85 of the 421 patients $(20.2 \%)$ had no follow-up examinations, 100 patients $(23.7 \%)$ had 1 follow-up examination, 80 patients $(19.0 \%)$ had 2 follow-up examinations, 68 patients $(16.2 \%)$ had 3 follow-up examinations, and 88 patients $(20.9 \%)$ had 4 or more follow-up examinations. Regarding the clinical course of the 47 patients who met criteria for depression 3 months after stroke, 37 of those 47 patients $(78.7 \%)$ were evaluated at least once following that baseline assessment. The mean change in SIGH-D total score between that baseline assessment and each patient's final assessment was a decrease of $6.62 \pm 5.85$ points. The magnitude of change ranged from a decrease of 18 points to an increase of 4 points. Thirty of the 37 patients $(81.1 \%)$ who met criteria for depression in that baseline assessment exhibited a decrease of 1 or more points and 13 of those 37 patients (35.1\%) exhibited a decrease of 10 or more points. Twenty of those 37 patients $(54.0 \%)$ never again received a SIGH-D total 
score greater than our cut-off for depression, 6 patients (16.2\%) remained depressed at all subsequent assessments, 3 patients $(8.1 \%)$ were again found to be depressed at a subsequent assessment but not at their final assessment, 5 patients $(13.5 \%)$ were not depressed at a subsequent assessment but were depressed at their final assessment, and 3 patients $(8.1 \%)$ exhibited an erratic course. Regarding the clinical course of the 374 patients who did not meet criteria for depression 3 months after stroke, 299 of those 374 patients $(80.0 \%)$ were evaluated at least once following that baseline assessment. Thirty-seven of those 299 patients (12.4\%) met criteria for depression at least once during long-term follow-up, while 262 of those patients $(87.6 \%)$ never met criteria for depression.

\section{SIGH-D Item Endorsement by Stroke and Dementia Status}

We compared the stroke and control cohorts with regard to the frequencies of endorsement of each of the items on the SIGH-D during the assessment that was performed 3 months after stroke. Chi-square analyses suggested that stroke status was associated with positive responses to the SIGH-D items referring to depressed mood, anergia, decreased appetite, weight loss, suicidal ideation, somatic complaints possibly associated with anxiety, hypochondriasis, and psychomotor retardation. Logistic regression determined that stroke status was independently associated with anergia (OR: 1.70; CI: 1.20-2.42), weight loss (OR: 1.82; CI: 1.05-3.17), hypochondriasis (OR: 1.82; CI: $1.25-$ 2.64), and psychomotor retardation (OR: 3.20; CI: 1.40-7.34).

Three months after stroke, demented patients differed from nondemented patients with regard to SIGH-D total score $(6.8 \pm 5.7$ vs. $4.3 \pm 4.3, p<.001$ by $t$ test $)$, the frequency of depression defined as a total score over 11 (23.2\% vs. $7.3 \%, p<.001$ by chi-square), and the frequency of depression defined as a total score over 11 and the spontaneous acknowledgment of depressed mood (15.2\% vs. $4.7 \%, p<.001$ by chi-square). Regarding dementia subtypes, patients with vascular dementia did not differ significantly from patients with Alzheimer's disease with concomitant stroke with regard to SIGH-D total score or the frequencies of depression recognized using our two paradigms.

We compared the demented and nondemented stroke patients with regard to the frequencies of endorsement of each of the items on the SIGH-D. Chi-square analyses suggested that dementia status was associated with positive responses to the SIGH-D items referring to depressed mood, decreased participation in work and activities, decreased appetite, weight loss, early and late insomnia, suicidal ideation, feelings of tension and irritability, hypochondriasis, lack of insight, and psychomotor retardation. Logistic regression determined that dementia status was independently associated with decreased participation in work and activities (OR: 2.08; CI: 1.04-4.15), weight loss (OR: 1.66; CI: .91-3.00), late insomnia (OR: 2.20; CI: 1.21-4.00), and psychomotor retardation (OR: 2.07; CI: 1.02-4.18).

\section{DISCUSSION}

We identified depression in $11.2 \%$ of our cohort 3 months after ischemic stroke. When depression was present in our stroke cohort, it tended to be mild, and in most cases it remitted in annual follow-up examinations. Depression was associated with a major hemispheral stroke syndrome, reflecting the severity of neurologic impairment; infarcts in the ACA and PCA territories, which supply limbic structures; dementia; and female sex. Interestingly, we found that the odds ratios for those first two variables were higher while the odds ratio for dementia was lower in a multivariate model of the determinants of depression identified using our more restrictive paradigm, suggesting that more severe strokes involving limbic structures are associated with depressed mood. When stroke patients were compared with stroke-free control subjects and demented stroke patients with nondemented stroke patients in the full cohort, however, the SIGH-D items that were most discriminating were those that referred to somatic symptoms, while depressed mood was not related to either stroke status or dementia status.

Most prior studies have reported higher frequencies of post-stroke depression than our own, but those higher frequencies were themselves quite variable, as were the determinants that have been identified (Gordon \& Hibbard, 1997). The studies of Robinson and his colleagues have been particularly influential. In one early study (Robinson et al. 1983), for example, they found that $27 \%$ of a sample of 103 stroke patients had major depression and $20 \%$ had dysthymic disorder using DSM-III criteria. Based on studies focusing primarily on patients with hemispheral involvement, Robinson and his colleagues have suggested that the location of the presenting lesion is an important determinant of the frequency and severity of post-stroke depression, with anterior lesions involving the left hemisphere placing patients at greatest risk (Robinson et al., 1983, 1984; Starkstein et al., 1987). Among patients with right hemisphere stroke, they have found that posterior lesions are associated with post-stroke depression (Robinson et al., 1984; Starkstein et al., 1989). Despite the hypothesized importance of lesion location in the etiology of post-stroke depression, they have also reported that the symptom profiles of patients with post-stroke major depression and functional major depression are similar (Lipsey et al., 1986).

In studies performed by other groups, Kotila et al. (1998) diagnosed depression, defined as a Beck Depression Inventory score over 9 , in $47 \%$ of their cohort of patients 3 months after first stroke; House et al. (1991) diagnosed major depression or dysthymic disorder in $12 \%$ of their patients 1 month after first stroke and $8.5 \%$ of their control subjects based on DSM-III criteria; and other groups have reported intermediate frequencies of depression (Andersen et al., 1994a; Burvill et al., 1995; Dennis et al., 2000; Ebrahim 
et al., 1987; Herrmann et al., 1998; Kim \& Choi-Kwon, 2000; Pohjasvaara et al., 1998b; Wade et al., 1987). Although certain studies have confirmed a relationship between anterior lesions of the left hemisphere and depression (Åström et al., 1993; Sinyor et al., 1986a), most have not (Andersen et al., 1995; Angeleri et al., 1997; Dam et al., 1989; Gainotti et al., 1997a; Herrmann et al., 1998; House et al., 1990; Schwartz et al., 1993). Carson et al. (2000) performed a meta-analysis of 35 previous studies that had investigated lesion location as a risk factor for depression. They did not recognize a significant association between those characteristics, noting that the relative risk of depression was 0.95 (CI: .83-1.10) for a left hemisphere versus a right hemisphere lesion location and 1.17 (CI: .87-1.62) for a left anterior hemispheral lesion location versus all other hemispheral lesion locations. In our own study, we found that infarcts in the ACA and PCA territories were associated with an increased frequency of depression, consistent with the report of Grasso et al. (1994) on the remote effects of subcortical lesions on limbic structures, but hemispheric laterality and a frontal lesion location were not relevant. Alternatively, prior studies have recognized the importance of factors such as a premorbid history of affective illness (Andersen et al., 1995; Herrmann et al., 1998; $\mathrm{Ng}$ et al., 1995; Pohjasvaara et al., 1998b; Schwartz et al., 1993), post-stroke functional disability (Burvill et al., 1997; Dennis et al., 2000; Ebrahim et al., 1987; Herrmann et al., 1998; Ng et al., 1995; Pohjasvaara et al., 1998b; Sato et al., 1999; Schwartz et al., 1993; Sinyor et al., 1986b; van de Weg et al., 1999; Wade et al., 1987), and a lack of social support (Andersen et al., 1995; Åström et al., 1993; Burvill et al., 1997) as determinants of depression after stroke.

It has been suggested that post-stroke depression is a categorical rather than a continuous construct, with distinguishable determinants of its major and minor forms (Morris et al., 1994; Paradiso \& Robinson, 1999). In contrast, the unimodal rather than bimodal shape of the graph of total scores on the SIGH-D in our stroke cohort shown in the Figure and the results of a number of prior studies suggest that post-stroke depression is a matter of degree rather than kind. That is, both major and minor post-stroke depression appear to be primarily due to psychological reactions to the consequences of stroke (Gainotti et al., 1997b, 1999), particularly functional disability (Burvill et al., 1997; Dennis et al., 2000; Ebrahim et al., 1987; Herrmann et al., 1998; $\mathrm{Ng}$ et al., 1995; Pohjasvaara et al., 1998b; Sato et al., 1999; Schwartz et al., 1993; Sinyor et al., 1986b; van de Weg et al., 1999; Wade et al., 1987), or endogenous factors such as a premorbid history of affective illness (Andersen et al., 1995; Herrmann et al., 1998; Ng et al., 1995; Pohjasvaara et al., 1998b; Schwartz et al., 1993), while the relevance of characteristics such as lesion location is less clear. The frequency of depression that we recognized in our stroke cohort is one of the lowest that has been reported, and, as suggested by the results of certain studies cited above, we propose that our findings were influenced primarily by the relatively low frequency of premorbid psychiatric illness that was present in our cohort. A premorbid history of depression was reported in $22 \%$ of the patients of Andersen et al. (1994a) and 19\% of the patients of Pohjasvaara et al. (1998b), for example, but only $5.7 \%$ of our stroke cohort had a premorbid history of any significant psychiatric illness. A psychiatric history was more common among patients with than without depression in our study (i.e., 8.5\% vs. $5.4 \%$ ), but that difference was not statistically significant due to the underrepresentation of that characteristic and a lack of statistical power.

Although the frequency of depression that we reported was relatively low, the true frequency of depression in our cohort may have been even lower due to incorrect classifications of depression resulting from the high frequency of somatic complaints related to stroke and/or dementia but not depression. Specifically, when we applied our alternative paradigm, in which we additionally required the spontaneous acknowledgment of depressed mood, the frequency of depression in our stroke cohort dropped from $11.2 \%$ to $7.4 \%$. Robinson and his colleagues have suggested that somatic symptoms are not more common among stroke patients unless depressed mood is also present (Fedoroff et al., 1991; Paradiso et al., 1997), but our findings suggest that clinicians should be sensitive to the similarities between the somatic features that are commonly seen in older medical patients and the clinical features that are conventionally accepted to be typical in depression (Linden et al., 1995 ) and rely more on nonsomatic than somatic symptoms in order to derive the most accurate diagnoses (Stein et al., 1996).

Our study has certain limitations. First, our study was performed at a tertiary care center, and most of our patients were admitted to a dedicated stroke unit and treated by neurologists specializing in stroke. It is likely that those factors contributed to better neurologic outcomes and a reduced frequency of depression. Second, we could have performed our baseline assessments in the acute phase following stroke, which might have resulted in a higher estimate of the frequency of depression, but we performed those assessments 3 months after stroke in order to examine patients in a more stable state following the period of maximal recovery. We did note that 21 of the 374 patients who did not meet criteria for depression 3 months after stroke (5.6\%) were taking anti-depressant medications, however, suggesting that some of those patients might have met criteria for depression if we had assessed them before that treatment was initiated. Similarly, in certain cases, rehabilitation programs may have enhanced physical recovery and thus mood during the first 3 months after stroke, suggesting that earlier assessments could have identified additional cases of depression prior to that intervention. Third, patients who were not examined 3 months after stroke were more severely impaired neurologically than patients who were examined, and the exclusion of those patients could have resulted in an underestimation of the frequency of depression. In addition, patients who were not examined 3 months after stroke were older and more often had a history of 
congestive heart failure than patients who were examined, reducing our ability to identify associations between those variables and depression. Fourth, the SIGH-D has certain limitations as a screening instrument, including its focus on symptomatology occurring during the preceding week, which could result in the failure to identify cases of depression that are fluctuating or that have recently begun to remit, and its weighting toward somatic complaints, which could cause the overestimation of the frequency of depression among older, neurologically impaired patients. As reviewed in detail in another publication (Desmond et al., 2000), it would have been optimal for us to administer a clinical interview such as the Structured Clinical Interview for DSM-IV Axis I Disorders (First et al., 1997) for the diagnosis of depression, and alternative measures such as the Geriatric Depression Scale (Yesavage et al., 1983) are also available. Fifth, we, like other groups, did not recognize an association between lesion location and depression, which may have resulted in part from our failure to use quantitative brain imaging measures in our analyses. We did note that variables representing lesion lateralization and frontal versus nonfrontal lesion locations were not relevant, however, suggesting that more precise measures should not have produced different results.

Finally, it is important to note that very few of our depressed patients were being treated for that disorder, consistent with the findings of numerous previous studies (Angeleri et al., 1997; Ebrahim et al., 1987; Robinson \& Price, 1982; Wade et al., 1987). Regardless of the absolute frequency of depression after stroke, it is a treatable disorder that warrants intervention when recognized. The results of the limited clinical trials that have been performed in stroke samples suggest that pharmacologic treatment can be effective (Rigler, 1999; Robinson, 1998).

\section{ACKNOWLEDGMENTS}

Presented in part at the 26th International Stroke Conference of the American Heart Association; Fort Lauderdale, Florida; February 15, 2001. This work was supported by Grants R01-NS26179 (Dr. Desmond), P01-AG07232 (Richard Mayeux, MD), K07AG00959 (Suzanne Mirra, MD), and K08-NS02051 (Dr. Moroney) from the National Institutes of Health. The authors would like to acknowledge the staff of the Stroke and Aging Research Project for their assistance with data collection.

\section{REFERENCES}

Agrell, B. \& Dehlin, O. (1989). Comparison of six depression rating scales in geriatric stroke patients. Stroke, 20, 1190-1194.

American Psychiatric Association. (1987). Diagnostic and statistical manual of mental disorders (Rev. 3rd ed.). Washington, DC: American Psychiatric Association.

Andersen, G., Vestergaard, K., Ingemann-Nielsen, M., \& Lauritzen, L. (1995). Risk factors for post-stroke depression. Acta Psychiatrica Scandinavica, 92, 193-198.

Andersen, G., Vestergaard, K., \& Lauritzen, L. (1994b). Effective treatment of poststroke depression with the selective serotonin reuptake inhibitor citalopram. Stroke, 25, 1099-1104.

Andersen, G., Vestergaard, K., Riis, J.Ø., \& Lauritzen, L. (1994a). Incidence of post-stroke depression during the first year in a large unselected stroke population determined using a valid standardized rating scale. Acta Psychiatrica Scandinavica, 90, 190-195.

Angeleri, F., Angeleri, V.A., Foschi, N., Giaquinto, S., Nolfe, G., Saginario, A., \& Signorino, M. (1997). Depression after stroke: An investigation through catamnesis. Journal of Clinical Psychiatry, 58, 261-265.

Åström, M., Adolfsson, R., \& Asplund, K. (1993). Major depression in stroke patients. A 3-year longitudinal study. Stroke, 24, 976-982.

Blessed, G., Tomlinson, B.E., \& Roth, M. (1968). The association between quantitative measures of dementia and of senile change in the cerebral grey matter of elderly subjects. British Journal of Psychiatry, 114, 797-811.

Bolla-Wilson, K., Robinson, R.G., Starkstein, S.E., Boston, J., \& Price, T.R. (1989). Lateralization of dementia of depression in stroke patients. American Journal of Psychiatry, 146, 627-634.

Burvill, P.W., Johnson, G.A., Jamrozik, K.D., Anderson, C.S., Stewart-Wynne, E.G., \& Chakera, T.M.H. (1995). Prevalence of depression after stroke: the Perth Community Stroke Study. British Journal of Psychiatry, 166, 320-327.

Burvill, P.W., Johnson, G.A., Jamrozik, K.D., Anderson, C.S., \& Stewart-Wynne, E.G. (1997). Risk factors for post-stroke depression. International Journal of Geriatric Psychiatry, 12, 219-226.

Carson, A.J., MacHale, S., Allen, K., Lawrie, S.M., Dennis, M., House, A., \& Sharpe, M. (2000). Depression after stroke and lesion location: A systematic review. Lancet, 356, 122-126.

Dam, H., Pedersen, H.E., \& Ahlgren, P. (1989). Depression among patients with stroke. Acta Psychiatrica Scandinavica, 80, 118-124.

Dennis, M., O’Rourke, S., Lewis, S., Sharpe, M., \& Warlow, C. (2000). Emotional outcomes after stroke: Factors associated with poor outcome. Journal of Neurology, Neurosurgery, and Psychiatry, 68, 47-52.

Desmond, D.W. (2000). The evaluation of mood and behavior in patients with focal brain lesions. In J. Bogousslavsky \& J.L. Cummings (Eds.), Behavior and mood disorders in focal brain lesions (pp. 21-47). Cambridge, UK: Cambridge University Press.

Desmond, D.W., Moroney, J.T., Paik, M.C., Sano, M., Mohr, J.P., Aboumatar, S., Tseng, C.L., Chan, S., Williams, J.B.W., Remien, R.H., Hauser, W.A., \& Stern, Y. (2000). Frequency and clinical determinants of dementia after ischemic stroke. Neurology, 54, 1124-1131.

Ebrahim, S., Barer, D., \& Nouri, F. (1987). Affective illness after stroke. British Journal of Psychiatry, 151, 52-56.

Endicott, J., Cohen, J., Nee, J., Fleiss, J., \& Sarantakos, S. (1981). Hamilton Depression Rating Scale. Extracted from Regular and Change Versions of the Schedule for Affective Disorders and Schizophrenia. Archives of General Psychiatry, 38, 98-103.

Fedoroff, J.P., Starkstein, S.E., Parikh, R.M., Price, T.R., \& Robinson, R.G. (1991). Are depressive symptoms nonspecific in patients with acute stroke? American Journal of Psychiatry, 148, 1172-1176.

First, M.B., Spitzer, R.L., Gibbon, M., \& Williams, J.B.W. (1997). User's guide for the Structured Clinical Interview for DSM-IV Axis I Disorders-Clinician Version (SCID-CV). Washington, DC: American Psychiatric Press. 
Folstein, M.F., Folstein, S.E., \& McHugh, P.R. (1975). "Minimental state": A practical method for grading the cognitive state of patients for the clinician. Journal of Psychiatric Research, 12, 189-198.

Foulkes, M.A., Wolf, P.A., Price, T.R., Mohr, J.P., \& Hier, D.B. (1988). The Stroke Data Bank: Design, methods, and baseline characteristics. Stroke, 19, 547-554.

Gainotti, G., Azzoni, A., Gasparini, F., Marra, C., \& Razzano, C. (1997a). Relation of lesion location to verbal and nonverbal mood measures in stroke patients. Stroke, 28, 2145-2149.

Gainotti, G., Azzoni, A., \& Marra, C. (1999). Frequency, phenomenology and anatomical-clinical correlates of major poststroke depression. British Journal of Psychiatry, 175, 163-167.

Gainotti, G., Azzoni, A., Razzano, C., Lanzillotta, M., Marra, C., \& Gasparini, F. (1997b). The Post-Stroke Depression Rating Scale: A test specifically devised to investigate affective disorders of stroke patients. Journal of Clinical and Experimental Neuropsychology, 19, 340-356.

Goodglass, H. \& Kaplan, E. (1983). The assessment of aphasia and related disorders (2nd ed.). Philadelphia: Lea \& Febiger.

Gordon, W.A. \& Hibbard, M.R. (1997). Poststroke depression: An examination of the literature. Archives of Physical Medicine and Rehabilitation, 78, 658-663.

Grasso, M.G., Pantano, P., Ricci, M., Intiso, D.F., Pace, A., Padovani, A., Orzi, F., Pozzilli, C., \& Lenzi, G.L. (1994). Mesial temporal cortex hypoperfusion is associated with depression in subcortical stroke. Stroke, 25, 980-985.

Hamilton, M. (1960). A rating scale for depression. Journal of Neurology, Neurosurgery, and Psychiatry, 23, 56-62.

Hamilton, M. (1967). Development of a rating scale for primary depressive illness. British Journal of Social and Clinical Psychology, 6, 278-296.

Herrmann, N., Black, S.E., Lawrence, J., Szekely, C., \& Szalai, J.P. (1998). The Sunnybrook Stroke Study: A prospective study of depressive symptoms and functional outcome. Stroke, 29, 618-624.

House, A., Dennis, M., Mogridge, L., Warlow, C., Hawton, K., \& Jones, L. (1991). Mood disorders in the year after first stroke. British Journal of Psychiatry, 158, 83-92.

House, A., Dennis, M., Warlow, C., Hawton, K., \& Molyneux, A. (1990). Mood disorders after stroke and their relation to lesion location. A CT scan study. Brain, 113, 1113-1129.

Kim, J.S. \& Choi-Kwon, S. (2000). Poststroke depression and emotional incontinence: Correlation with lesion location. $\mathrm{Neu}$ rology, 54, 1805-1810.

Kotila, M., Numminen, H., Waltimo, O., \& Kaste, M. (1998). Depression after stroke. Results of the FINNSTROKE Study. Stroke, 29, 368-372.

Linden, M., Borchelt, M., Barnow, S., \& Geiselmann, B. (1995). The impact of somatic morbidity on the Hamilton Depression Rating Scale in the very old. Acta Psychiatrica Scandinavica, 92, 150-154.

Lipsey, J.R., Spencer, W.C., Rabins, P.V., \& Robinson, R.G. (1986). Phenomenological comparison of poststroke depression and functional depression. American Journal of Psychiatry, 143, 527-529.

Mahoney, F.I. \& Barthel, D.W. (1965). Functional evaluation: The Barthel Index. Maryland Medical Journal, 14, 61-65.

Malec, J.F., Richardson, J.W., Sinaki, M., \& O’Brien, M.W. (1990). Types of affective response to stroke. Archives of Physical Medicine and Rehabilitation, 71, 279-284.

Morris, P.L.P., Robinson, R.G., Andrzejewski, P., Samuels, J., \& Price, T.R. (1993). Association of depression with 10-year poststroke mortality. American Journal of Psychiatry, 150, 124-129.

Morris, P.L.P., Shields, R.B., Hopwood, M.J., Robinson, R.G., \& Raphael, B. (1994). Are there two depressive syndromes after stroke? Journal of Nervous and Mental Disease, 182, 230-234

Ng, K.C., Chan, K.L., \& Straughan, P.T. (1995). A study of poststroke depression in a rehabilitative center. Acta Psychiatrica Scandinavica, 92, 75-79.

Paradiso, S., Ohkubo, T., \& Robinson, R.G. (1997). Vegetative and psychological symptoms associated with depressed mood over the first two years after stroke. International Journal of Psychiatry in Medicine, 27, 137-157.

Paradiso, S. \& Robinson, R.G. (1999). Minor depression after stroke: An initial validation of the DSM-IV construct. American Journal of Geriatric Psychiatry, 7, 244-251.

Parikh, R.M., Robinson, R.G., Lipsey, J.R., Starkstein, S.E., Fedoroff, J.P., \& Price, T.R. (1990). The impact of poststroke depression on recovery in activities of daily living over a 2-year follow-up. Archives of Neurology, 47, 785-789.

Pohjasvaara, T., Erkinjuntti, T., Vataja, R., \& Kaste, M. (1998a). Correlates of dependent living 3 months after ischemic stroke. Cerebrovascular Diseases, 8, 259-266.

Pohjasvaara, T., Leppävuori, A., Siira, I., Vataja, R., Kaste, M., \& Erkinjuntti, T. (1998b). Frequency and clinical determinants of poststroke depression. Stroke, 29, 2311-2317.

Rigler, S.K. (1999). Management of poststroke depression in older people. Clinics in Geriatric Medicine, 15, 765-783.

Robinson, R.G. (1998). Treatment issues in poststroke depression. Depression and Anxiety, 8, 85-90.

Robinson, R.G., Kubos, K.L., Starr, L.B., Rao, K., \& Price, T.R. (1984). Mood disorders in stroke patients. Importance of location of lesion. Brain, 107, 81-93.

Robinson, R.G \& Price, T.R. (1982). Post-stroke depressive disorders: A follow-up study of 103 patients. Stroke , 13, 635-641.

Robinson, R.G., Starr, L.B., Kubos, K.L., \& Price, T.R. (1983). A two-year longitudinal study of post-stroke mood disorders: Findings during the initial evaluation. Stroke, 14, 736-741.

Román, G.C., Tatemichi, T.K., Erkinjuntti, T., Cummings, J.L., Masdeu, J.C., Garcia, J.H., Amaducci, L., Orgogozo, J.M., Brun, A., Hofman, A., Moody, D.M., O’Brien, M.D., Yamaguchi, T., Grafman, J., Drayer, B.P., Bennett, D.A., Fisher, M., Ogata, J., Kokmen, E., Bermejo, F., Wolf, P.A., Gorelick, P.B., Bick, K.L., Pajeau, A.K., Bell, M.A., DeCarli, C., Culebras, A., Korczyn, A.D., Bogousslavsky, J., Hartmann, A., \& Scheinberg, P. (1993). Vascular dementia: Diagnostic criteria for research studies. Report of the NINDS-AIREN International Workshop. Neurology, 43, 250-260.

Sarti, C., Rastenyte, D., Cepaitis, Z., \& Tuomilehto, J. (2000). International trends in mortality from stroke, 1968 to 1994. Stroke, 31, 1588-1601.

Sato, R., Bryan, R.N., \& Fried, L.P. (1999). Neuroanatomic and functional correlates of depressed mood: The Cardiovascular Health Study. American Journal of Epidemiology, 150, 919-929.

Schwartz, J.A., Speed, N.M., Brunberg, J.A., Brewer, T.L., Brown, M., \& Greden, J.F. (1993). Depression in stroke rehabilitation. Biological Psychiatry, 33, 694-699.

Sinyor, D., Amato, P., Kaloupek, D.G., Becker, R., Goldenberg, M., \& Coopersmith, H. (1986b). Post-stroke depression: Relationships to functional impairment, coping strategies, and rehabilitation outcome. Stroke, 17, 1102-1107.

Sinyor, D., Jacques, P., Kaloupek, D.G., Becker, R., Goldenberg, M., \& Coopersmith, H. (1986a). Poststroke depression and 
lesion location. An attempted replication. Brain, 109, 537-546.

Starkstein, S.E., Robinson, R.G., Honig, M.A., Parikh, R.M., Joselyn, J., \& Price, T.R. (1989). Mood changes after righthemisphere lesions. British Journal of Psychiatry, 155, 79-85.

Starkstein, S.E., Robinson, R.G., \& Price, T.R. (1987). Comparison of cortical and subcortical lesions in the production of poststroke mood disorders. Brain, 110, 1045-1059.

Stein, P.N., Sliwinski, M.J., Gordon, W.A., \& Hibbard, M.R. (1996). Discriminative properties of somatic and nonsomatic symptoms for post stroke depression. Clinical Neuropsychologist, 10, 141-148.

Stern, Y., Andrews, H., Pittman, J., Sano, M., Tatemichi, T., Lantigua, R., \& Mayeux, R. (1992). Diagnosis of dementia in a heterogeneous population: Development of a neuropsychological paradigm-based diagnosis of dementia and quantified cor- rection for the effects of education. Archives of Neurology, 49 $453-460$

van de Weg, F.B., Kuik, D.J., \& Lankhorst, G.J. (1999). Poststroke depression and functional outcome: A cohort study investigating the influence of depression on functional recovery from stroke. Clinical Rehabilitation, 13, 268-272.

Wade, D.T., Legh-Smith, J., \& Hewer, R.A. (1987). Depressed mood after stroke. A community study of its frequency. British Journal of Psychiatry, 151, 200-205.

Williams, J.B.W. (1988). A structured interview guide for the Hamilton Depression Rating Scale. Archives of General Psychiatry, 45, 742-747.

Yesavage, J.A., Brink, T.L., Rose, T.L., Lum, O., Huang, V., Adey, M., \& Leirer, V.O. (1983). Development and validation of a geriatric depression screening scale: A preliminary report. Journal of Psychiatric Research, 17, 37-49. 